\title{
THE SIGNIFICANT CYLINDRICAL CONDITION AND A HAUSDORFF RECTIFIABLE SET
}

\author{
J. C. BRECKENRIDGE
}

\begin{abstract}
Area measures of the Geöcze type can be constructed for continuous parametric surfaces of finite Lebesgue area provided the surface satisfies the significant cylindrical condition. It is shown that requiring this condition is equivalent to requiring the Hausdorff rectifiability of a certain subset of the range of the surface.
\end{abstract}

Introduction. The construction of area measures of the Geöcze type for continuous $k$-dimensional parametric surfaces in $n$-space uses not only the basic hypothesis of finiteness of area but, when $2<k<n$, additional hypotheses as well. Thus the surface may be required to (a) belong to the lower area quasi additivity class or, equivalently, (b) satisfy the significant cylindrical condition (see [8]). These two hypotheses are known to be weaker than requiring the $(k+1)$-dimensional Hausdorff measure of the range of the surface to vanish.

The purpose of this note is to show (Theorem 2.1) that if the surface has finite Lebesgue $k$-area, then hypothesis (b) is equivalent to requiring the Hausdorff $k$-rectifiability of a certain subset of its range.

1. Preliminaries. Let $f: X \rightarrow R^{n}$ be a continuous mapping of finite Lebesgue $k$-area from a compact oriented $k$-dimensional $C^{\infty}$ manifold $X$ with $2 \leqq k \leqq n$, and let $A$ be a subset of $X$.

$\Lambda(n, k)$ will denote the set of all integer $k$-tuples $\lambda=\left(\lambda_{1}, \cdots, \lambda_{k}\right)$ with $1 \leqq \lambda_{1}<\cdots<\lambda_{k} \leqq n$. Corresponding to each such $\lambda$, let $p_{\lambda}$ be the orthogonal projection of $R^{n}$ onto $R^{k}$ defined by $p_{\lambda}\left(y_{1}, \cdots, y_{n}\right)=\left(y_{\lambda_{1}}, \cdots, y_{\lambda_{k}}\right)$, and set $f^{\lambda}=p_{\lambda} \circ f$. The restriction of $f$ [resp., $f^{\lambda}$ ] to a subset $E$ of $X$ will be denoted by $(f, E)$ [resp., $\left(f^{i}, E\right)$ ], and $L$ will denote the Lebesgue $k$-area functional. Thus, $L(f, E)=\sup L(f, T)$, where the supremum is taken over all finitely triangulable subsets $T$ of $E$.

1.1. The commutative diagram

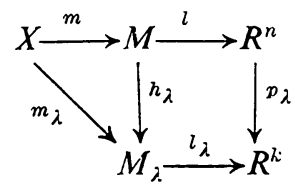

Received by the editors February 29, 1972 and, in revised form, May 12, 1972. AMS (MOS) subject classifications (1970). Primary 28 A75.

Key words and phrases. Surface area, area measures.

(C) American Mathematical Society 1973 
represents the monotone-light factorizations, $f=l \circ m$ and $f^{\lambda}=l_{\lambda} \circ m_{\lambda}$, of $f$ and $f^{\lambda}$ through their respective middle spaces $M$ and $M_{\lambda}$. It follows from [4] (see also [7]) that $L\left(f^{\lambda}, A\right)=L\left(f^{\lambda}, A_{\lambda}\right)$, where $A_{\lambda}$ denotes the union of all maximal continua of constancy for $f^{\lambda}$ contained in the interior of $A$. Hence, by [5] (see also [7], [8], [2]), the formula

$$
\bar{\mu}_{\lambda}(B)=\inf L\left(f^{\lambda}, A \cap m_{\lambda}^{-1}(G)\right) \quad\left(B \subset M_{\lambda}\right),
$$

where the infimum is taken over all open sets $G$ in $M_{i}$ containing $B$, defines a finite Borel measure $\bar{\mu}$, on $M_{\lambda}$ for which there are Borel sets $E_{\lambda}$ satisfying

$$
\begin{aligned}
& E_{\lambda} \subset m_{\lambda}(A)-m_{\lambda}\left(A^{*}\right) \subset M_{\lambda}, \\
& \bar{\mu}_{\lambda}\left(M_{\lambda}-E_{\lambda}\right)=0, \\
& L_{k}\left[l_{\lambda}\left(B \cap E_{\lambda}\right)\right]=0 \quad \text { whenever } B \subset M_{\lambda} \text { and } \bar{\mu}_{\lambda}(B)=0 .
\end{aligned}
$$

Here, $A^{*}$ denotes the frontier of $A$, and $L_{k}$ denotes the $k$-dimensional Lebesgue measure on $R^{k}$.

1.2. Let $C_{j}$ be the $F_{\sigma}$-subset of $M$ on which $h_{j}$ fails to be univalent. Then $(f, A)$ is said to satisfy the significant cylindrical condition (briefly, SCC) if $\bar{\mu}_{\lambda}\left[h_{i}\left(C_{\lambda}\right)\right]=0$ for each $\lambda \in \Lambda(n, k)$. This condition is necessary and sufficient in order that the formulas

$$
\mu_{\dot{\lambda}}(B)=\inf L\left(f^{i}, A \cap m^{-1}(G)\right) \quad(B \subset M),
$$

where the infima are taken over all open sets $G$ in $M$ containing $B$, define finite Borel measures $\mu_{;}$, on $M$ satisfying

$$
\bar{\mu}_{i}(B)=\mu_{i}\left[h_{i}^{-1}(B)\right] \quad\left(B \subset M_{i}\right),
$$

for each $\lambda \in \Lambda(n, k)$. (See [8].)

Whenever $(f, A)$ satisfies the SCC, $\mu$ will denote the total variation measure (with respect to the Euclidean norm) of the vector measure $\left[\mu_{j}: \lambda \in \Lambda(n, k)\right]$. Thus $\mu$ is the lower $k$-area measure of $(f, A)$. (See [8], [1].)

1.3. $H_{n}^{k}$ will denote the $k$-dimensional Hausdorff measure on $R^{n}$. Corresponding to each $\lambda \in \Lambda(n, k)$ and each subset $E$ of $R^{n}$, let

$$
\begin{aligned}
& H_{\lambda}(E)=\inf H_{n}^{k}(E-N), \\
& D_{\lambda}(E)=\left\{y \in R^{n}: \limsup _{r \rightarrow 0^{+}} H_{\lambda}[E \cap s(y, r)] / r^{k}>0\right\},
\end{aligned}
$$

where the infimum is taken over all $H_{n}^{k}$-measurable subsets $N$ of $R^{n}$ for which $L_{k}\left[p_{i}(N)\right]=0$, and where $s(y, r)$ denotes the open ball in $R^{n}$ with center $y$ and radius $r . H_{j}$ is a metric outer measure on $R^{n}$, and $D_{\dot{\lambda}}(E)$ is a Borel set. 
Corresponding to each $\lambda \in \Lambda(n, k)$, let $E_{\lambda}$ be a Borel set satisfying (2), and define Borel subsets $S_{\lambda}=S_{\lambda}(f, A)$ and $S=S(f, A)$ of $M$ as follows:

$$
\begin{aligned}
S_{\lambda} & =h_{\lambda}^{-1}\left(E_{\lambda}\right) \cap l^{-1}\left\{D_{\lambda}\left[\left(l \circ h_{\lambda}^{-1}\right)\left(E_{\lambda}\right)\right]\right\}, \\
S & =\bigcup_{\lambda \in \Lambda(n, k)} S_{\lambda} .
\end{aligned}
$$

These sets were first studied with $(k, n)=(2,3)$ by T. Radó [9].

1.4. THEOREM. If $H_{n}^{k}[l(S)]<\infty$, then $(f, A)$ satisfies the SCC.

1.5. THEOREM. If $(f, A)$ satisfies the $S C C$, then

(a) $\mu_{\lambda}\left(M-S_{\dot{\lambda}}\right)=0$ for each $\lambda \in \Lambda(n, k)$,

(b) $\mu(M-S)=0$;

further, if there exists a Borel subset $F$ of $M$ satisfying both $\mu(M-F)=0$ and $H_{n}^{k}[l(F)]<\infty$, then

(c) $H_{n}^{k}[l(S)]<\infty$,

(d) $H_{n}^{k}[l(B \cap S)]=0$ whenever $B \subset M$ and $\mu(B)=0$.

The proofs are the same as given in [2].

2. The rectifiability of $l(S)$. Recall that a subset $W$ of $R^{n}$ is $H_{n^{-}}^{k}$ rectifiable if and only if $H_{n}^{k}(W)<\infty$ and $H_{n}^{k}$-almost all of $W$ is contained in the union of a countable family of $k$-dimensional $C^{1}$ submanifolds of $R^{n}$ [6, p. 267].

2.1. THEOREM. ( $f, A)$ satisfies the $S C C$ if and only if the set $l(S)$, $S=S(f, A)$, is $H_{n}^{k}$-rectifiable.

Sufficiency follows from (1.4). The proof of necessity will follow from (1.5) together with results from [5]. Notations concerning currents and differential forms are those of [5] and [6].

Corresponding to each $C^{\infty}$ mapping $f_{i}: X \rightarrow R^{n}$, let $v_{i}$ be the current valued measure on $M$ defined by

$$
v_{i}(g)(\varphi)=\int_{\lambda^{-}}(g \circ m) \wedge f_{i}^{\#}(\varphi)
$$

for each continuous real valued function $g$ on $M$ and each $C^{\infty}$ differential $k$-form $\varphi$ on $R^{n}$. By $[5,3.4]$ there is a sequence of $C^{\infty}$ mappings $f_{i}$ that converge uniformly to $f$, whose Lebesgue $k$-areas are bounded, and whose associated measures $v_{i}$ converge weakly to a current valued Borel measure $v$ on $M$; moreover, $v$ satisfies the conditions of the representation theorem [5, 2.1-2.2]. In particular, there is a nonnegative integer valued Baire function $|v|$ on $M$ such that

$$
\|v\|(B)=\int_{R^{n}} \sum_{z \in B \cap l^{-1}(y)}|v(z)| d H_{n}^{k}(y)<\infty
$$


for every Borel subset $B$ of $M$. (Here, $\|v\|$ denotes the total variation measure of $v$ with respect to mass.) Also, if $F=\{z \in M:|v(z)| \neq 0\}$, then $l(F)$ is $H_{n}^{k}$-rectifiable.

It follows from [5, 3.6, 4.1], (1), and (2) that if $B$ is a Borel subset of $M_{\lambda}$, then $\bar{\mu}_{\lambda}(B) \leqq\|v\|\left[h_{\lambda}^{-1}(B)\right]$; hence, since $(f, A)$ satisfies the SCC, and by (3),

$$
\mu_{\lambda}(B)=\mu_{\lambda}\left(B-C_{\lambda}\right)=\bar{\mu}_{\lambda}\left[h_{\lambda}\left(B-C_{\lambda}\right)\right] \leqq\|v\|(B)
$$

for every Borel subset $B$ of $M$ and every $\lambda \in \Lambda(n, k)$. Thus $\mu$ is absolutely continuous with respect to $\|v\|$ and, by (4), $\mu(M-F)=0$. Finally, since $l(F)$ is $H_{n}^{k}$-rectifiable, it follows from (1.5) that $H_{n}^{k}$-almost all of $l(S)$ is contained in $l(F)$ and, consequently, that $l(S)$ is $H_{n}^{k}$-rectifiable.

2.2. REMARK. The preceding theorem also applies, for example, to any continuous mapping $g: Q \rightarrow R^{n}$ of finite Lebesgue $k$-area from a $k$-cell $Q$ in $R^{k}$, provided $(g, Q)$ satisfies the SCC. In fact, by the method of doubling, there is a homeomorph $A$ of $Q$ contained in the $k$-sphere $S^{k}$ and a continuous mapping $f: S^{k} \rightarrow R^{n}$ of finite Lebesgue $k$-area such that $(f, A)$ and $(g, Q)$ are Lebesgue equivalent.

\section{REFERENCES}

1. J. C. Breckenridge, Cesari-Weierstrass surface integrals and lower k-area, Ann. Scuola Norm. Sup. Pisa (2) 25 (1971), 423-446.

2. - Geöcze $k$-area and significant sets, Accad. Naz. Sci. Lett. Arti Modena Atti Mem. (to appear).

3. L. Cesari, Surface area, Ann. of Math. Studies, no. 35, Princeton Univ. Press, Princeton, N.J., 1956. MR 17, 596.

4. H. Federer, Essential multiplicity and Lebesgue area, Proc. Nat. Acad. Sci. U.S.A. 34 (1948), 611-616. MR 10, 361.

5. —, Currents and area, Trans. Amer. Math. Soc. 98 (1961), 204-233. MR 23 \#A1006.

6. - Geometric measure theory, Die Grundlehren der math. Wissenschaften, Band 153, Springer-Verlag, New York, 1969. MR 41 \#1976.

7. T. Nishiura, Integrals over a product variety and Fubini theorems, Rend. Circ. Mat. Palermo (2) 14 (1965), 207-236. MR 33 \#5848.

8. - Area measure and Radó's lower area, Trans. Amer. Math. Soc. 159 (1971), 355-367.

9. T. Radó, On multiplicity functions associated with Lebesgue area, Rend. Circ. Mat. Palermo (2) 4 (1955), 219-236. MR 17, 595.

Department of Mathematics, Wayne State University, Detroit, Michigan 48202 